\title{
Associations between Ambulatory Blood Pressure Parameters and Cerebral White Matter Lesions
}

\author{
Cristina Sierra \\ Hypertension Unit, Department of Internal Medicine, Institute of Medicine and Dermatology, IDIBAPS, \\ Hospital Clinic of Barcelona, University of Barcelona, Villarroel 170, 08036 Barcelona, Spain \\ Correspondence should be addressed to Cristina Sierra, csierra@clinic.ub.es
}

Received 6 April 2011; Accepted 31 May 2011

Academic Editor: Zafar Israili

Copyright ( $) 2011$ Cristina Sierra. This is an open access article distributed under the Creative Commons Attribution License, which permits unrestricted use, distribution, and reproduction in any medium, provided the original work is properly cited.

Silent cerebral white matter lesions (WMLs) are a common finding on magnetic resonance imaging of the brain in the elderly. However, in patients with hypertension, WMLs tend to occur earlier in life and appear to be more severe. There is a body of evidence that supports the idea that WMLs in asymptomatic hypertensive patients should be considered a silent early marker of brain damage. It is known that ambulatory blood pressure monitoring (ABPM) correlates more closely with hypertension-related organ damage than office blood pressure. This paper focuses on the associations between blood pressure parameters obtained by 24-hour ABMP and cerebral WMLs.

\section{Introduction}

On ageing, a nonmodifiable cardiovascular risk factor, hypertension becomes the most important modifiable risk factor for developing cerebrovascular disease, including stroke, cerebral small vessel disease (lacunar infarcts, white matter lesions, microbleeds), and cognitive impairment or vascular dementia.

Silent cerebral white matter lesions (WMLs) are a common finding on brain magnetic resonance imaging (MRI) in the elderly. However, in patients with hypertension, WMLS tend to occur earlier in life and appear to be more severe. There is a body of evidence that supports the idea that WML in asymptomatic hypertensive patients should be considered a silent early marker of brain damage. Cerebral WMLS are an important prognostic factor for stroke, cognitive impairment, dementia, and death [1]. Although the pathogenesis of cerebral WML remains controversial, older age and hypertension are constantly reported to be the main risk factors [2, 3] (Figure 1). Hypertensive patients have a higher rate and extension of cerebral WML compared with normotensives $[2,4]$. In addition, it has been shown that treated, controlled hypertensive patients have a lower prevalence of WML than both untreated and treated but not controlled hypertensive patients [4]. Data from interventional and prospective observational studies also suggest that appropriate antihypertensive treatment could efficiently prevent the development of WML and slow their progression $[5,6]$.

Twenty-four hour ambulatory blood pressure monitoring (ABPM) has become an important tool for improving the diagnostic and management of hypertension. It is known that ABPM more closely correlates with hypertension-related organ damage and has a closer association with cardiovascular events than office blood pressure (BP) [7]. The information provided by 24-hour ABPM includes daytime and nighttime BP profiles, day-night BP difference, morning blood pressure rise, and blood pressure variability. Studies have found associations between 24-hour ABPM parameters and hypertensive target organ damage, such as left ventricular hypertrophy, microalbuminuria, intima media thickness, retinal changes, pulse wave velocity, and silent brain damage (lacunar infarct, WML) (Figure 2) $[8,9]$. We review current evidence on the relationship between 24-hour ABPM parameters and WML.

\section{Relationship between 24-Hour, Daytime, and Nighttime ABP Values and Cerebral WML}

The association between hypertension and WML has been established in cross-sectional $[2,4,10-12]$ and longitudinal 


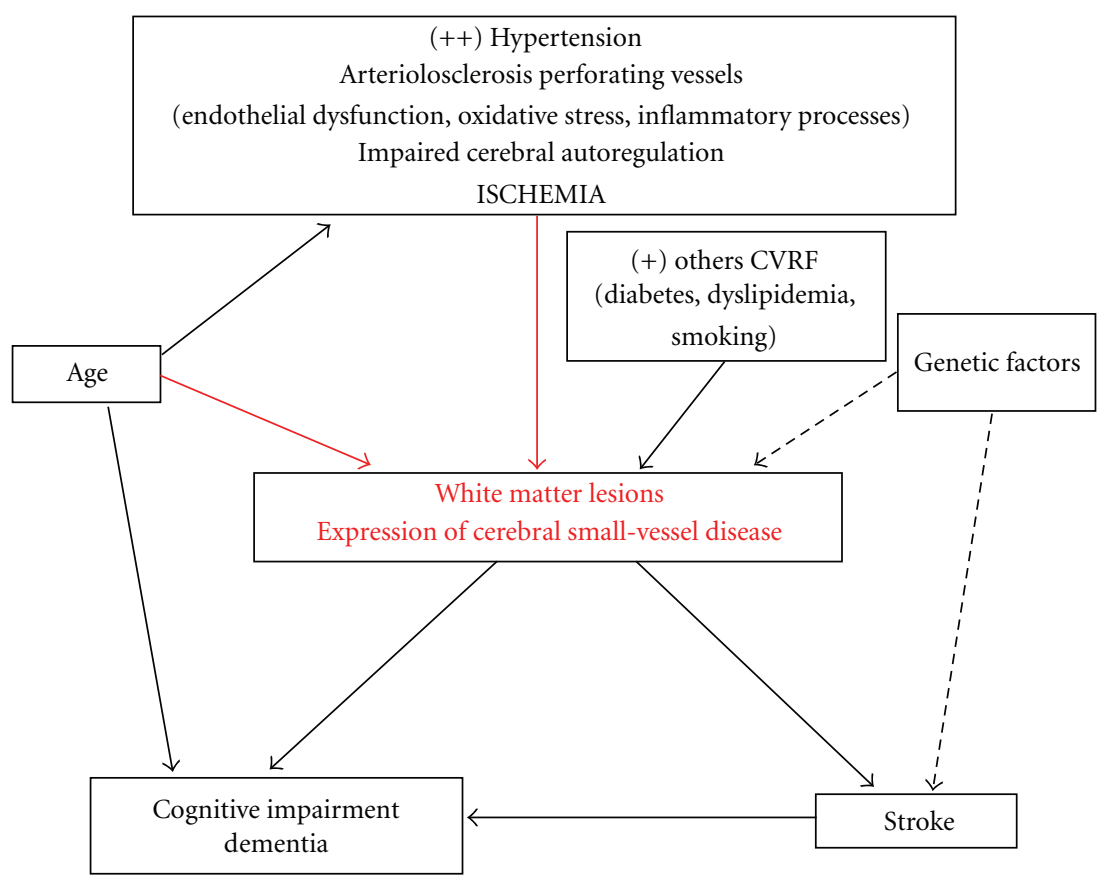

FIGURE 1: Pathogenesis and clinical significance of cerebral white matter lesions.

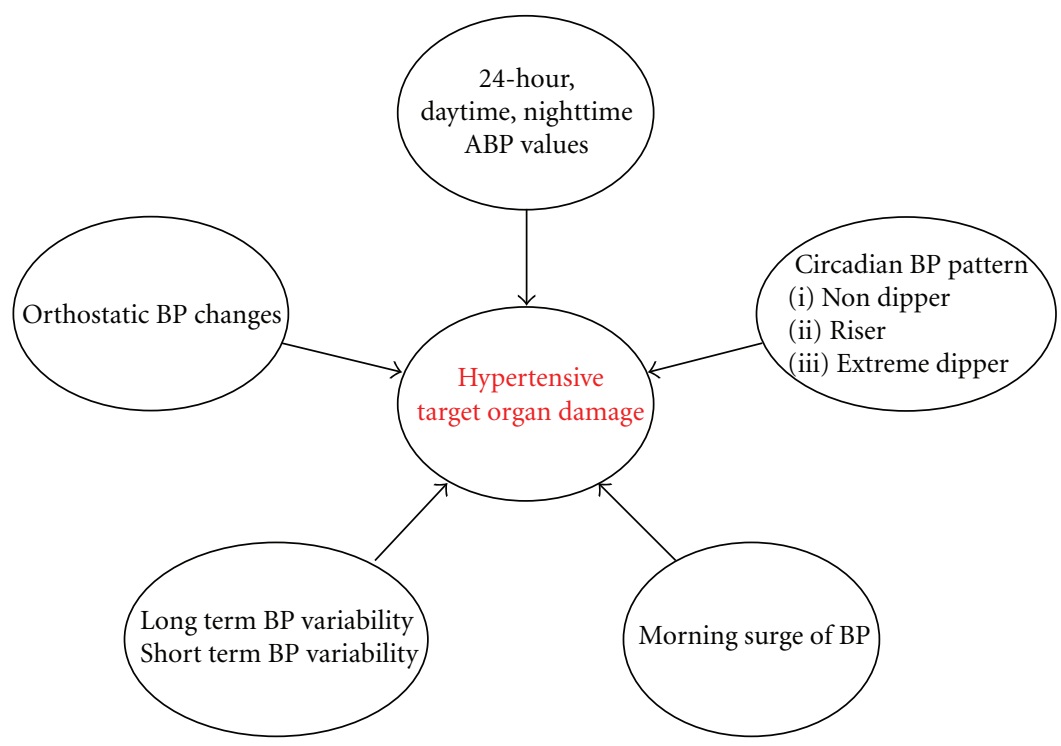

FiguRE 2: Associations among different ambulatory blood pressure parameters and hypertensive target organ damage.

studies [13-15]. However, some reports have suggested that this relationship is only evident when 24-hour ABPM is used to assess BP. Goldstein et al. [16] found a correlation between WML and office systolic, but not diastolic, BP, in a group of elderly normotensive subjects. Conversely, the severity of WML correlated with both systolic and diastolic BP measured by ABPM. In a group of mixed normotensives, "white coat" hypertensives, and sustained hypertensives, Shimada et al. [12] found a correlation between the number of lacunae and periventricular hyperintensities with 24hour BP, but not with office BP. Sierra et al. [17] found a correlation between WML and both clinic BP and 24-hour $\mathrm{ABPM}$ in 66 untreated middle-aged hypertensive patients. In this study also higher BP values (including office, 24hour, daytime were and nighttime estimates) in hypertensive patients with WML compared with those without [17] found.

In a recent study of 210 asymptomatic hypertensive patients (mean age: $52.5 \pm 12.5$ years) it was found that higher 24-hour, daytime, and nighttime BP levels were independently associated with WML volume (MRI semiautomatic volume quantification) [18]. WML volume and ABP levels, 
whether daytime, nighttime, or 24-h, were continuous, without evidence of distinct thresholds, and continued down to $\mathrm{BP}$ levels within the normotensive range. The authors suggest that this dose effect of BP on WML, though cross-sectional, is supportive of a causal relationship between increasing BP levels and the development of WML.

One longitudinal study assessed the BP-WML relationship using ABPM [19] in 155 healthy elderly individuals (range: 55-79 years; mean age: 66.2) who were followed for five years, at which time a second 24-hour ABPM was performed in 121 subjects. The initial cross-sectional findings showed that in a sample of healthy subjects with relatively low BP levels (mean: 116.9/71.1 $\mathrm{mmHg}$ ), men and women with casual BP in the upper ranges had a higher WML severity rating. Subjects whose casual or waking systolic BP remained high for five years relative to the group were more likely to exhibit higher WML volume during both phases of the study than the remaining study subjects, especially those whose initial low BP remained low.

Some studies have also shown an association between higher PP values (including office and ambulatory 24-hour, daytime, and nighttime estimates), a measure of arterial stiffness, and WML $[17,20]$. In addition, it has recently been shown that brachial BP is associated with WML in the elderly [21].

\section{Circadian Blood Pressure Patterns and Cerebral WML}

The natural circadian BP rhythm typically includes a nocturnal decrease of $10-20 \%$ in BP compared with daytime, waking values. However, there is a moderate-to-marked loss of this reduction in nighttime BP in between 25\% and 35\% of hypertensive patients, a phenomenon that has been associated with excessive cardiac, vascular, renal, and cerebrovascular target organ damage [22].

Yamamoto et al. [23] studied 105 patients with lacunar infarcts who were followed for $3.2 \pm 2.6$ years and found that a high mean ambulatory BP, especially nighttime BP, and a reduced nocturnal BP dip, adversely affected the development of silent ischemic lesions (lacunar infarcts and WML) and symptomatic stroke.

In the aforementioned study by Goldstein et al. [16] of 144 healthy elderly individuals aged 55-79 years, it was found that subjects with the highest WML severity rating had higher casual, waking, and sleeping systolic BP, higher waking diastolic BP, higher waking systolic BP variability, and a smaller nocturnal fall in systolic and diastolic BP than individuals with less severe WML ratings. Sander et al. [24] studied 227 healthy subjects aged $>55$ years $(44 \%$ hypertensive; $12 \%$ diabetic patients) and found that subjects with WML were significantly older, had a greater frequency of a history of hypertension, and had an elevated mean systolic daytime BP, a reduced systolic circadian BP variation, and an increased incidence of pathological nighttime BP increases. They found a significant correlation between systolic circadian BP variation and the extent of WML. Multiple regression analysis showed that this parameter was best correlated with the extent of WML. Kario et al. [25], in a study of 131 elderly hypertensives (aged $\geq 60$ years), found that both nondippers and extreme dippers had significantly more silent cerebrovascular damage (measuring both lacunar infarcts and WML) than dippers. Similarly, Shimada et al. [26], who studied asymptomatic elderly hypertensives, observed no differences in cerebral abnormalities (lacunar infarct and WML) between the normotensive group and the dipping hypertensive group. However, hypertensive dippers had higher ambulatory BP than normotensive individuals and a larger BP reduction from day to night than both the normotensive and hypertensive nondipping groups. These findings suggest that a dipping profile may inhibit the development of cerebrovascular abnormalities.

One reason why marked nocturnal BP fall may be associated with cerebrovascular disease is that the lower BP limit of BP in the self-regulation of cerebral blood flow is shifted upward, especially in elderly hypertensive patients with brain damage. Marked nighttime BP falls could lead to an excessive reduction in cerebral perfusion. In the study by Kario et al. [25], some patients had been treated before the study, and a greater fall in nocturnal BP due to antihypertensive medication might have accelerated brain ischemia. Atherosclerosis may also be a link between excess nocturnal BP fall and cerebrovascular damage. In the same study [25], although both nondippers and extreme dippers suffered more extensive cerebrovascular damage (lacunar infarcts and WML) than dippers, there were no significant differences between extreme dippers and dippers in terms of cardiac hypertrophy and renal damage, whereas these types of target organ damage were more frequent in nondippers than in dippers. It appears that nondippers show greater hypertensive target organ damage than extreme dippers do. Therefore, as the authors suggest, sustained high BP over prolonged periods seems to be the most important determinant of hypertensive end-organ damage, whereas marked nocturnal BP falls may be more specifically related to cerebrovascular damage. Birns et al. [27] studied 88 hypertensive patients (mean age: 65 years) who were on antihypertensive therapy and optimally controlled, with preexisting hypertensive cerebrovascular disease (WML) and without a history of stroke, transient ischemic attack or syncope in the previous three months. Mean daytime/nighttime systolic BP values were $136.2 \mathrm{mmHg}$ and $127.8 \mathrm{mmHg}$, respectively, and mean daytime/nighttime diastolic BP values were $77.7 \mathrm{mmHg}$ and $71.1 \mathrm{mmHg}$, respectively. In the study it was found that a physiological fall in nighttime BP in these hypertensives was associated with greater WML volume which, in turn, correlated with impairments in reaction time and verbal fluency. However, the cross-sectional design could not establish causality, and therefore further studies are necessary.

In contrast, circadian rhythms were not related to WML in a small group of 66 middle-aged (mean age: 54 years) never-treated hypertensive patients [17], while in a group of 86 newly diagnosed hypertensive individuals (mean age 57.4 years; range $40-80$ ), no relationship between diurnal BP rhythm and WML was found [20].

It is clear that the relationships between office, waking, and sleeping BP and cerebral vascular disease vary according to patient characteristics (age, normotensive/hypertensive, 
asymptomatic/clinical cerebrovascular disease), the methodology used to evaluate WML volumes, and whether patients are receiving antihypertensive drug therapy. However, a body of evidence supports the idea that, in addition to increased daytime BP, raised sleeping BP and abnormal nocturnal BP reduction are associated with more severe WML.

The studies mentioned have not determined whether nondipping is the cause or consequence of cerebrovascular disease. Reductions in nocturnal BP falls might be secondary to site-specific cerebral injuries resulting in impaired central autonomic nervous system functioning. Kario and Shimada [28] presented a 79-year-old hypertensive patient whose diurnal BP pattern changed after a minor ischemic stroke (small lacunar infarct), suggesting that abnormal diurnal BP might originate from minor cerebrovascular ischemia. Some reports have shown that the level and variation of $\mathrm{BP}$ and/or heart rate might change in patients with overt cerebrovascular disease [29]. Korpelainen et al. [29] studied 40 brain infarction patients and 5 healthy controls and found that, in addition to increased sympathetic activity, brain infarction also seems to cause parasympathetic hypofunction. Goldstein et al. [16] investigated diurnal BP variation and subcortical MRI-T2 hyperintensities in 144 healthy individuals aged 55 to 79 years. Individuals with a higher prevalence of hyperintensities in the insular subcortex, which plays an important role in cerebral self-regulation, had higher 24-hour BP. In a study of a homogeneous sample of never-treated hypertensive patients aged 50-60 years, after exclusion for known risk factors for cerebrovascular damage such as diabetes or significant alcohol intake, it was found that subjects with WML had a blunted fall in nocturnal heart rate compared with those without WML [17].

Although some data seem to suggest that chronic ischemia caused by hypertension leads to disrupted diurnal $\mathrm{BP}$ variations through the impairment of cerebral self-regulation, resulting in nondipping in sleeping BP, most studies have been cross-sectional and, therefore, the direction of the relationship observed between some parameters and WML remains unclear. Larger, longitudinal studies will be needed to establish causality.

\section{Blood Pressure Variability and Cerebral WML}

Although BP variability has been associated with target organ damage in hypertension [30], the relationship with cerebral alterations remains unclear. Goldstein et al. [16] suggested a higher standard deviation of waking systolic BP in patients with more severe WML. In the aforementioned study by Kario et al. [25], it was found that extreme dippers had greater BP variability (standard deviation of waking systolic $\mathrm{BP}$ ) and more WML and lacunar infarcts than dippers.

In one study it was found that asymptomatic middleaged hypertensives with WML had significantly higher values of long-term systolic BP variability (standard deviation of 24hour BP) measured by continuous beat-to-beat monitoring and ABPM, compared to those without WML. However, the differences were not independent of BP elevation and the significance was not maintained after adjustment for 24hour systolic BP. There were no differences in short-term systolic BP variability or short-term or long-term diastolic BP variability in patients with and without WML [31]. Shortterm BP variability was obtained by calculating the mean standard deviations of mean systolic and diastolic BP values for each 48 half-hour period (within half-hour standard deviation). Long-term variability was calculated by obtaining the mean 48 half-hour systolic and diastolic BP mean values and calculating the standard deviation of the mean (among half-hour standard deviation).

The two major determinants of BP variability are age and high BP, which are also major cardiovascular risk factors. Therefore, the significant impact of BP variability on cardiovascular disease seems to depend partly on age and high BP. Puisieux et al. [32] retrospectively reviewed computed tomography scans and 24-hour ABPM in 79 patients (mean age: 83 years) and found that higher WML scores were associated with increased blood pressure variability. To evaluate short-term BP variation, they determined the variability of systolic and diastolic BP (within-subject standard deviation of all readings over a 24-hour period), the coefficient of variability (variability of $\mathrm{BP} /$ mean $\mathrm{BP}$ ), and the maximal $\mathrm{BP}$ variation (difference between the maximum and minimum 24-hour BP). Higher WML scores were associated with higher systolic BP in 24-hour, diurnal and nocturnal periods, higher maximal variation of systolic BP, greater variability of 24-hour diurnal and nocturnal systolic BP, and a greater coefficient of variability of systolic BP during sleep. They concluded that elevations and short-term variations in systolic BP may contribute to the pathogenesis of WML in the elderly. However, 50 of the 79 patients were suffering from dementia (30 Alzheimer's disease and 18 vascular dementia).

The longitudinal epidemiological Honolulu-Asia Aging Study of the risk factors for cardiovascular disease showed that midlife office systolic BP variability was associated with WML detected in late life in 585 males [33]. Excess systolic $\mathrm{BP}$ variability was defined as greater than average increases in BP measurements from up to 3 examinations over 6 years. The mechanism for these relationships is unknown. The authors suggest that a possible explanation could involve chronic periods of higher and lower systolic BP levels that overcome the self-regulation that maintains the blood flow in the cranial vessels. This variability could result in periods of relative ischemia in vulnerable areas. In fact, the deeper white matter tissues are supplied by terminal vessels in the brain and lack sufficient anastomoses with other vessels, and these tissues might be at higher risk from systolic BP variation.

\section{Orthostatic Blood Pressure Changes and WML}

In healthy subjects, BP variation due to posture-dependent changes is minimal due to self-regulation. In most hypertensive patients without autonomic nervous dysfunction, BP posture-dependent changes are also minimal. Orthostatic hypotension, often found in elderly hypertensives, is recognized as a risk for falls, syncope, cardiovascular events, and death [34-38]. 
Matsubayashi et al. [39] studied 334 community-dwelling adults aged $>75$ years and found that both postural hypotension and postural hypertension were closely related to WML and poorer neurobehavioral function scores. Subjects with more advanced WML had exaggerated postural changes in BP. Postural hypotension was defined as a reduction in systolic $\mathrm{BP} \geq 20 \mathrm{mmHg}$ and postural hypertension as an increase in systolic BP $\geq 20 \mathrm{mmHg}$ using differences between the mean of two measurements of systolic BP while subjects were standing and supine, respectively.

Kario et al. [40] performed a head-up tilting test on elderly subjects with sustained hypertension as indicated by 24-hour ABPM. They were classified as having orthostatic hypertension (orthostatic increase in systolic BP of $\geq 20 \mathrm{mmHg}(n=26)$ ), orthostatic hypotension (orthostatic systolic BP reduction of $\geq 20 \mathrm{mmHg}(n=23)$ ), or being normal (neither pattern $(n=192))$. The results showed that silent lacunar infarcts were more common in patients with orthostatic hypotension and hypertension than in normal subjects. Patients with orthostatic hypotension and hypertension had significantly greater BP variability (standard deviation of waking systolic BP) than normal subjects. The associations between orthostatic BP change and silent cerebrovascular disease remained significant after controlling for confounders, including ambulatory BP values.

These U-shaped associations between orthostatic BP changes and the prevalence of silent lacunar infarctions are consistent with recent data from the prospective, populationbased ARIC study [41], in which it was found that orthostatic systolic BP and diastolic BP reductions were associated with an increased incidence of thrombotic and cardioembolic strokes in a linear fashion. In addition, both orthostatic systolic BP reductions and increases were associated with an increased incidence of lacunar strokes.

Postprandial hypotension has also been related to silent WML. Kohara et al. [42] evaluated BP changes after a meal by 24 -hour ABPM in 70 hospitalized essential hypertensive patients aged $\geq 50$ years. They found that the prevalence and severity of both lacunar infarcts and WML were significantly related to postprandial hypotension.

\section{Morning Surge in Blood Pressure and Silent Cerebrovascular Disease}

Normal morning BP surge is a physiological phenomenon, but an exaggerated morning BP surge is a cardiovascular risk. The association between the degree of morning BP surge and cardiovascular risk is not linear but rather has a threshold [9]. Cross-sectional studies have shown associations between target organ damage (left ventricular hypertrophy, microalbuminuria, intima media thickness, pulse wave velocity, silent lacunar infarcts) and morning surge in BP [9]. Vascular diseases of both the small and large arteries are considered to be not only consequences but also the leading cause of exaggerated morning BP surge, giving rise to a vicious cycle in the cardiovascular continuum [9]. Different pressor factors (aging, hypertension, glucose abnormality, alcohol intake, smoking, psychological stress, physical stress) are associated with morning BP surge. Diurnal variation and activation of neurohumoral factors that regulate the vascular tonus and cardiac output, such as the renin-angiotensin and sympathetic nervous systems, are suggested to be involved in diurnal BP variation and morning BP surge [9].

No studies have yet investigated the relationship between morning surge in BP and WML. However, in one study of 191 hypertensives (mean age: 76 years), an association between a higher morning surge in BP and silent lacunar infarcts, another form of hypertensive-cerebral small vessel disease [43] was found. Morning surge in BP was defined by subtracting mean systolic BP during the one hour with the lowest sleeping BP from mean systolic BP during the two hours after waking. Subjects were classified into 2 groups according to the morning surge in BP, with a cutoff value of $55 \mathrm{mmHg}$. The study compared the incidence of silent brain infarcts and overt stroke between the two groups and found that the group with a higher morning surge group showed a higher prevalence of silent brain infarcts after matching for age and 24-hour ambulatory BP (70\% versus 49\%; $P=0.01)$. In addition, patients were followed for a mean of 41 months, and a higher morning BP surge was associated with stroke risk independently of ambulatory BP, nocturnal BP falls, and silent infarct. In this study, $51 \%$ of the patients in the morning surge group were classified as extreme dippers. When a combination of dipping status (extremedippers, dippers, nondippers, and risers) and morning surge in BP was included in the same Cox regression analysis model, stroke risk was significantly associated with both morning surge in BP and with riser status [43]. In the model, extreme dipper status was not significantly associated with stroke risk independently of morning surge. The authors suggest that the fall in BP during the night appears to be of less importance than the morning surge. The mechanism underlying the increased stroke risk of extreme-dippers might depend on either an excessive morning surge of BP or on cerebral hypoperfusion due to low nocturnal BP.

\section{Conclusions}

On ageing, hypertension becomes the most important factor for cerebral WMLs, which are an important prognostic factor for stroke, cognitive impairment, dementia, and death. Strong evidence supports the idea that cerebral WMLs in hypertensive patients should be considered a silent early marker of brain damage.

The pathogenesis of WML remains unclear, but the main current hypothesis concerning the association between high BP and WML is that long-standing hypertension causes lipohyalinosis of the media and thickening of the vessel walls, with narrowing of the lumen of the small perforating arteries and arterioles that nourish the deep white matter. Low BP has also been reported to be a risk factor for WML.

As with other hypertensive target organ damage, ABPM 24-hour values also are related to the presence and severity of cerebral WML. In addition, in most studies an association between different forms of higher BP variability of BP and WML. However most of these studies have been crosssectional has been found and, therefore, the relationship observed between some parameters and the presence of 
WML is an association whose direction remains speculative. Larger, longitudinal studies will be required to establish causality.

Various mechanisms may be involved in the association between BP variability and target organ damage and cardiovascular disease. In addition to augmented mechanical stress on the cardiovascular system, leading to cardiovascular remodeling, increased variability of blood flow due to augmented BP variability increases sheer stress on endothelial cells, thereby advancing atherosclerosis, as does sheer stress-induced platelet activation at atherosclerotic stenotic sites. Neurohumoral activation, which is increased in subjects with increased BP variability, may also increase the risk of developing target organ damage and, therefore, cardiovascular disease.

\section{References}

[1] S. Debette and H. S. Markus, "The clinical importance of white matter hyperintensities on brain magnetic resonance imaging: systematic review and meta-analysis," The British Medical Journal, vol. 341, no. 7767, p. 288, 2010.

[2] L. Pantoni and J. H. Garcia, "The significance of cerebral white matter abnormalities 100 years after Binswanger's report," Stroke, vol. 26, no. 7, pp. 1293-1301, 1995.

[3] L. Pantoni, "Cerebral small vessel disease: from pathogenesis and clinical characteristics to therapeutic challenges," The Lancet Neurology, vol. 9, no. 7, pp. 689-701, 2010.

[4] D. Liao, L. Cooper, J. Cai et al., "Presence and severity of cerebral white matter lesions and hypertension, its treatment, and its control. The ARIC Study," Stroke, vol. 27, no. 12, pp. 22622270, 1996.

[5] C. Dufouil, J. Chalmers, O. Coskun et al., "Effects of blood pressure lowering on cerebral white matter hyperintensities in patients with stroke. The PROGRESS Magnetic resonance imaging substudy," Circulation, vol. 112, no. 11, pp. 16441650, 2005.

[6] O. Godin, C. Tzourio, P. Maillard, B. Mazoyer, and C. Dufouil, "Antihypertensive treatment and change in blood pressure are associated with the progression of white matter lesion volumes: the Three-City (3C)-Dijon Magnetic Resonance Imaging Study," Circulation, vol. 123, pp. 266-273, 2011.

[7] G. Mancia, G. De Backer, A. Dominiczak et al., "2007 Guidelines for the Management of Arterial Hypertension: the Task Force for the Management of Arterial Hypertension of the European Society of Hypertension (ESH) and of the European Society of Cardiology (ESC)," Journal of Hypertension, vol. 25, no. 6, pp. 1105-1187, 2007.

[8] G. Mancia and G. Parati, "The role of blood pressure variability in end-organ damage," Journal of Hypertension-Supplement, vol. 21, no. 6, pp. S17-S23, 2003.

[9] K. Kario, "Morning surge in blood pressure and cardiovascular risk: evidence and perspectives," Hypertension, vol. 56, no. 5, pp. 765-773, 2010.

[10] W. T. Longstreth, T. A. Manolio, A. Arnold et al., "Clinical correlates of white matter findings on cranial magnetic resonance imaging of 3301 elderly people. The Cardiovascular Health Study," Stroke, vol. 27, no. 8, pp. 1274-1282, 1996.

[11] M. M. Breteler, J. C. van Swieten, M. L. Bots et al., "Cerebral white matter lesions, vascular risk factors, and cognitive function in a population-based study: the Rotterdam Study," Neurology, vol. 44, no. 7, pp. 1246-1252, 1994.
[12] K. Shimada, A. Kawamoto, K. Matsubayashi, and T. Ozawa, "Silent cerebrovascular disease in the elderly. Correlation with ambulatory pressure," Hypertension, vol. 16, no. 6, pp. 692699, 1990.

[13] R. Schmidt, F. Fazekas, P. Kapeller, H. Schmidt, and H. P. Hartung, "MRI white matter hyperintensities. Three-year followup of the Austrian Stroke Prevention Study," Neurology, vol. 53, no. 1, pp. 132-139, 1999.

[14] C. Dufouil, A. de Kersaint-Gilly, V. Besancon et al., "Longitudinal study of blood pressure and white matter hyperintensities. The EVA MRI cohort," Neurology, vol. 56, no. 7, pp. 921926, 2001.

[15] F. E. De Leeuw, J. C. de Groot, M. Oudkerk et al., "Hypertension and cerebral white matter lesions in a prospective cohort study," Brain, vol. 125, no. 4, pp. 765-772, 2002.

[16] I. B. Goldstein, G. Bartzokis, D. B. Hance, and D. Shapiro, "Relationship between blood pressure and subcortical lesions in healthy elderly people," Stroke, vol. 29, no. 4, pp. 765-772, 1998.

[17] C. Sierra, A. de la Sierra, J. Mercader, E. Gómez-Angelats, A. Urbano-Márquez, and A. Coca, "Silent cerebral white matter lesions in middle-aged essential hypertensive patients," Journal of Hypertension, vol. 20, no. 3, pp. 519-524, 2002.

[18] L. H. Henskens, A. A. Kroon, R. J. van Oostenbrugge et al., "Associations of ambulatory blood pressure levels with white matter hyperintensity volumes in hypertensive patients," Journal of Hypertension, vol. 27, no. 7, pp. 1446-1452, 2009.

[19] I. B. Goldstein, G. Bartzokis, D. Guthrie, and D. Shapiro, "Ambulatory blood pressure and the brain. A 5-year followup," Neurology, vol. 64, no. 11, pp. 1846-1852, 2005.

[20] M. P. J. van Boxtel, L. H. G. Henskens, A. A. Kroon et al., "Ambulatory blood pressure, asymptomatic cerebrovascular damage and cognitive function in essential hypertension," Journal of Human Hypertension, vol. 20, no. 1, pp. 5-13, 2006.

[21] C. K. Kim, S. H. Lee, B. J. Kim, W. S. Ryu, and B. W. Yoon, "Age-independent association of pulse pressure with cerebral white matter lesions in asymptomatic elderly individuals," Journal of Hypertension, vol. 29, pp. 325-329, 2011.

[22] F. S. Routledge, J. A. McFetridge-Durdle, C. R. Dean, and Canadian Hypertension Society, "Night-time blood pressure patterns and target organ damage: a review," The Canadian Journal of Cardiology, vol. 23, no. 2, pp. 132-138, 2007.

[23] Y. Yamamoto, I. Akiguchi, K. Oiwa, M. Hayashi, and J. Kimura, "Adverse effect of nighttime blood pressure the outcome of lacunar infarct patients," Stroke, vol. 29, no. 3, pp. 570-576, 1998.

[24] D. Sander, K. Winbeck, J. Klingelhöfer, and B. Conrad, "Extent of cerebral white matter lesions is related to changes of circadian blood pressure rhythmicity," Archives of Neurology, vol. 57, no. 9, pp. 1302-1307, 2000.

[25] K. Kario, T. Matsuo, H. Kobayashi, M. Imiya, M. Matsuo, and K. Shimada, "Nocturnal fall of blood pressure and silent cerebrovascular damage in elderly hypertensive patients. Advanced silent cerebrovascular damage in extreme dippers," Hypertension, vol. 27, no. 1, pp. 130-135, 1996.

[26] K. Shimada, A. Kawamoto, K. Matsubayashi, M. Nishinaga, S. Kimura, and T. Ozawa, "Diurnal blood pressure variations and silent cerebrovascular damage in elderly patients with hypertension," Journal of Hypertension, vol. 10, no. 8, pp. 875878, 1992.

[27] J. Birns, R. Morris, J. Jarosz, H. Markus, and L. Kalra, “The structural and functional consequences of diurnal variations in blood pressure in treated patients with hypertensive 
cerebrovascular disease," Journal of Hypertension, vol. 27, no. 5, pp. 1042-1048, 2009.

[28] K. Kario and K. Shimada, "Change in diurnal blood pressure rhythm due to small lacunar infarct," The Lancet, vol. 344, no. 8916, p. 200, 1994.

[29] J. T. Korpelainen, K. A. Sotaniemi, K. Suominen, U. Tolonen, and V. V. Myllyla, "Cardiovascular autonomic reflexes in brain infarction,” Stroke, vol. 25, no. 4, pp. 787-792, 1994.

[30] A. Frattola, G. Parati, C. Cuspidi, F. Albini, and G. Mancia, "Prognostic value of 24-hour blood pressure variability," Journal of Hypertension, vol. 11, no. 10, pp. 1133-1137, 1993.

[31] E. Gómez-Angelats, A. de la Sierra, C. Sierra, G. Parati, G. Mancia, and A. Coca, "Blood pressure variability and silent cerebral damage in essential hypertension," The American Journal of Hypertension, vol. 17, no. 8, pp. 696-700, 2004.

[32] F. Puisieux, P. Monaca, D. Deplanque et al., "Relationship between leuko-araiosis and blood pressure variability in the elderly," The European Neurology, vol. 46, no. 3, pp. 115-120, 2001.

[33] R. J. Havlik, D. J. Foley, B. Sayer, K. Masaki, L. White, and L. J. Launer, "Variability in midlife systolic blood pressure is related to late-life brain white matter lesions. The HonoluluAsia Aging study," Stroke, vol. 33, no. 1, pp. 26-30, 2002.

[34] K. H. Masaki, I. J. Schatz, C. M. Burchfiel et al., "Orthostatic hypotension predicts mortality in elderly men: the Honolulu Heart Program," Circulation, vol. 98, no. 21, pp. 2290-2295, 1998.

[35] M. L. Eigenbrodt, K. M. Rose, D. J. Couper, D. K. Arnett, R. Smith, and D. Jones, "Orthostatic hypotension as a risk factor for stroke: the atherosclerosis risk in communities (ARIC) study, 1987-1996," Stroke, vol. 31, no. 10, pp. 2307-2313, 2000.

[36] K. M. Rose, M. L. Eigenbrodt, R. L. Biga et al., "Orthostatic hypotension predicts mortality in middle-aged adults: the Atherosclerosis Risk in Communities (ARIC) Study," Circulation, vol. 114, no. 7, pp. 630-636, 2006.

[37] A. A. Ejaz, A. Kazory, and M. E. Heinig, "24-hour blood pressure monitoring in the evaluation of supine hypertension and orthostatic hypotension," Journal of Clinical Hypertension, vol. 9, no. 12, pp. 952-955, 2007.

[38] T. Badia Farré, F. Formiga Perez, J. Almeda Ortega, A. Ferrer Feliu, S. Rojas-Farreras, and Grupo Octabaix, "Relationship between blood pressure and mortality at 4years of follow up in a cohort of individuals aged over 80years," Medicina Clínica, vol. 137, no. 3, pp. 97-103, 2011.

[39] K. Matsubayashi, K. Okumiya, T. Wada et al., "Postural dysregulation in systolic blood pressure is associated with worsened scoring on neurobehavioral function tests and leukoaraiosis in the older elderly living in a community," Stroke, vol. 28, no. 11, pp. 2169-2173, 1997.

[40] K. Kario, K. Eguchi, S. Hoshide et al., "U-curve relationship between orthostatic blood pressure change and silent cerebrovascular disease in elderly hypertensives: orthostatic hypertension as a new cardiovascular risk factor," Journal of the American College of Cardiology, vol. 40, no. 1, pp. 133-141, 2002.

[41] H. Yatsuya, A. R. Folsom, A. Alonso, R. F. Gottesman, and K. M. Rose, "Postural changes in blood pressure and incidence of ischemic stroke subtypes. The ARIC study," Hypertension, vol. 57, pp. 167-173, 2011.

[42] K. Kohara, Y. Jiang, M. Igase et al., "Postprandial hypotension is associated with asymptomatic cerebrovascular damage in essential hypertensive patients," Hypertension, vol. 33, no. 1, pp. 565-568, 1999.
[43] K. Kario, T. G. Pickering, Y. Umeda et al., "Morning surge in blood pressure as a predictor of silent and clinical cerebrovascular disease in elderly hypertensives: a prospective study," Circulation, vol. 107, no. 10, pp. 1401-1406, 2003. 


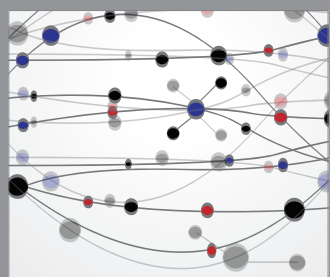

The Scientific World Journal
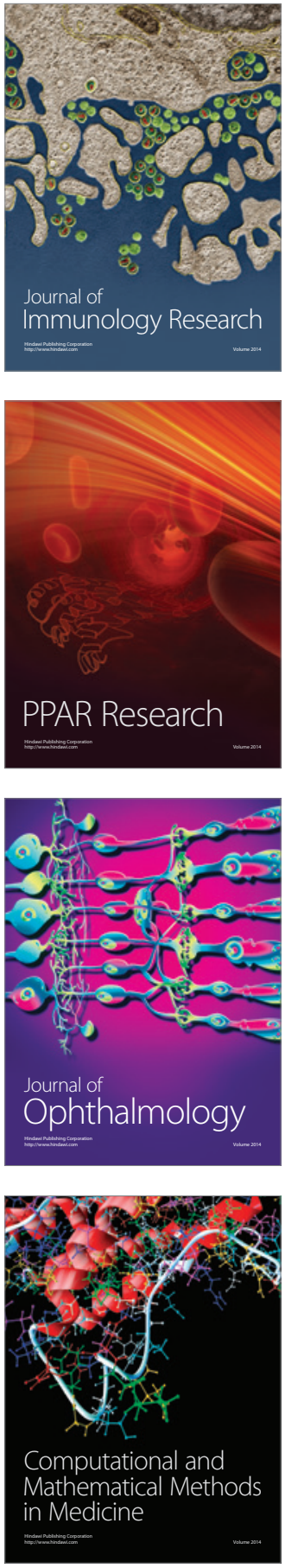

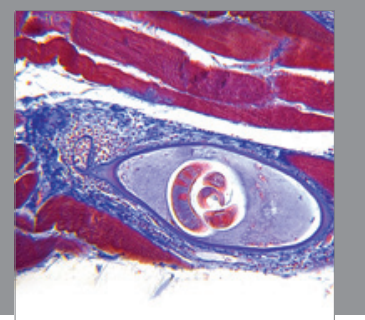

Gastroenterology

Research and Practice
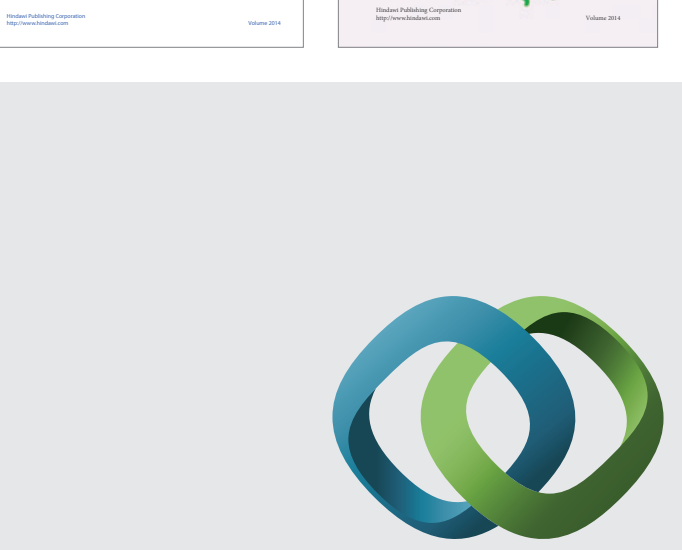

\section{Hindawi}

Submit your manuscripts at

http://www.hindawi.com
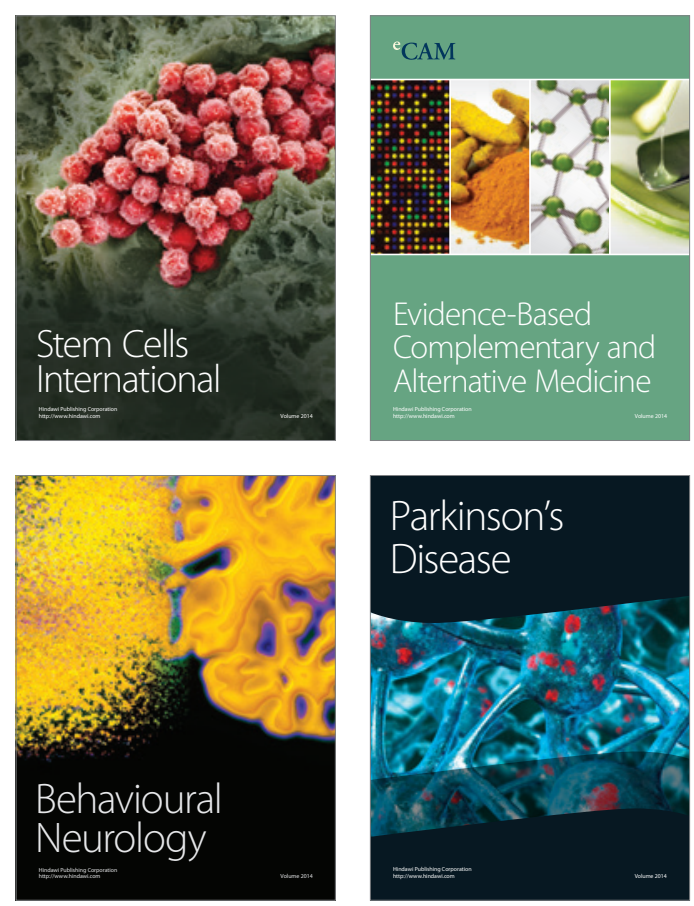

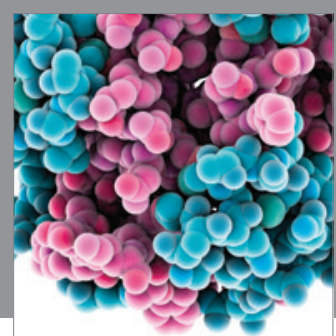

Journal of
Diabetes Research

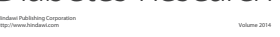

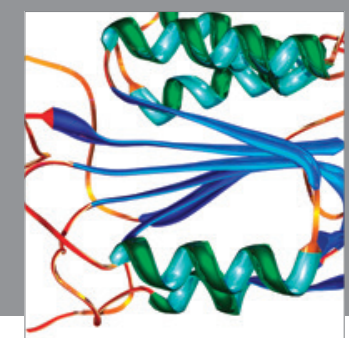

Disease Markers
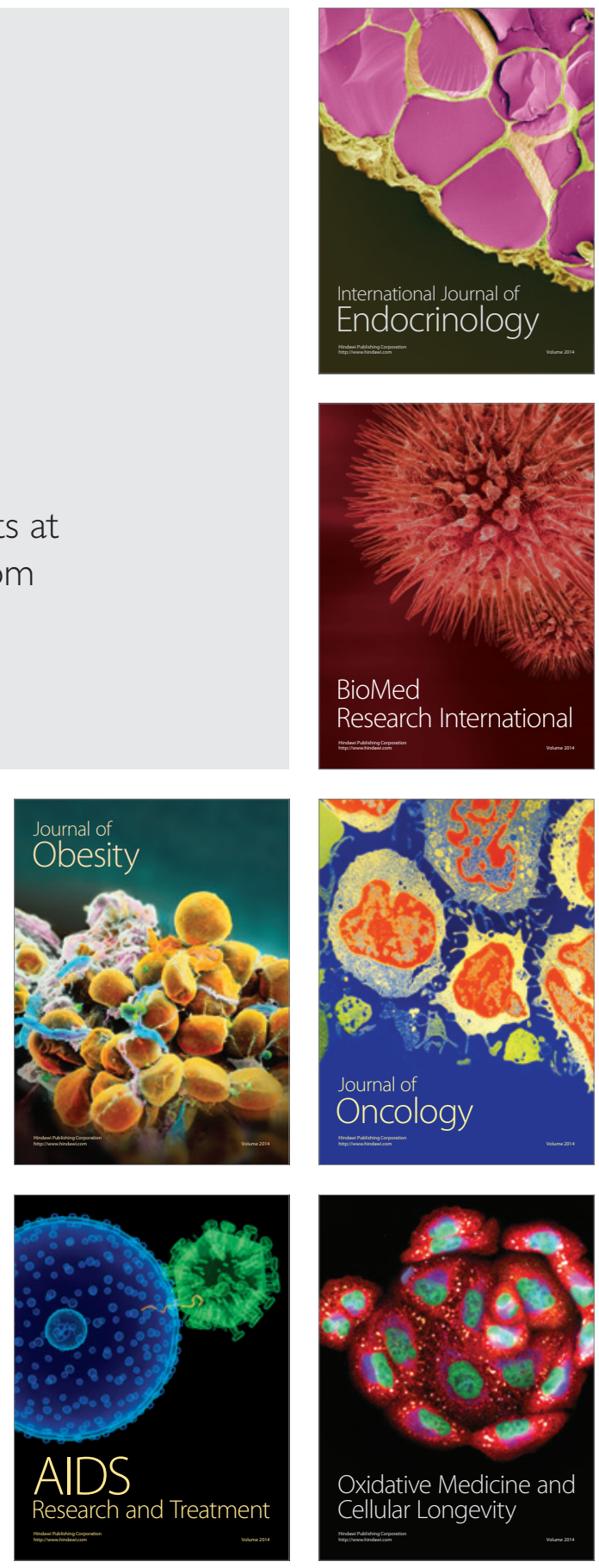